J. Sustain. Wireless Syst.

Vol.01/ No. 03

Pages: $148-160$

http://irojournals.com/irosws/

DOI: https://doi.org/10.36548/jsws.2019.3.002

\title{
ANALYSIS OF ROUTING PROTOCOLS IN FLYING WIRELESS NETWORKS
}

\author{
Mr. Krishnaraj Natarajan \\ Assistant Professor, \\ Department of IT, \\ Dr. Mahalingam College of Engineering \& Technology, \\ Pollachi, India. \\ Email id: krishna.rajtce@gmail.com
}

\begin{abstract}
The wireless network that are formed on the fly, without relying on the previous infrastructure, including the autonomous devices that lie nearby are called the flying wireless networks. Despite of the networks, self-configuring and selfhealing nature, it incurs many challenges due to its mobile nature, limited battery span and the lack of the centralized control, so these wireless network requires a perfect routing protocol addressing the challenges and improving the performance of the network, so the paper presents the survey on the various routing protocols that enhances the performance of the flying wireless network. The survey is further continued with the analysis of the routing protocols to evince the capability of the same on the grounds of network life time, energy consumption and the quality of service.
\end{abstract}

Keywords: Flying wireless networks, routing protocols, performance analysis, network life time, energy consumption and the quality of service.

\section{Introduction}

The tremendous improvement in the tele-communication and the information technology lead to the emerging of the wireless network that subdued the complex setting up of the wired networks, along with the complications involved in it, in the process of transmission. The wireless networks were more preferred than the wired networks, as they allow the transmission over the free space using the electromagnetic waves that elude the necessity of using the large bunch of wires that are draped everywhere. Moreover the wireless network operates over an entire network coverage covering more area, unlike the wired networks that operates within a limited area. This convenience of accessing the network resources from anywhere that is under your network coverage, the easy expandability with the available equipment's, the robust security protections, the ability to move along anywhere without the necessity of being tied to a place for the access of the network and the cost-effectiveness has made the wireless networks to be more favored than the wired networks. The wireless networks that are constructed using the access-points that control and host the devices in the wireless connection are called the managed networks or centralized networks that 
J. Sustain. Wireless Syst.

Vol.01/ No. 03

Pages: 148-160

http://irojournals.com/irosws/

DOI: https://doi.org/10.36548/jsws.2019.3.002

are framed with infrastructure, they are certain wireless networks that are not under the control of any access-points and are framed as network on the move without relying the infrastructure, such networks are called adhoc as they purely serve a particular purpose. The nodes or the data-points take up a dual-action and play the role of both the host that initiates the transmission and the router that forward the information to the next node [21]. They form the network with the nearby available nodes whenever a necessity arises for the transmission. The nodes or the data points that are used in forming the network might be homogenous, if the network is contained with the same type of devices or heterogeneous, if the device contained are different. The nodes in the engaged in the network could possess different characteristics in terms of storage, energy availability and processing capability. Any devices can volunteer itself or leave the network, at any time without any intimation as they are mobile. This autonomous behavior and the mobile nature of the devices, causes the human intervention in its configuration to be seldom. This wireless network that forms a network without an managing point and transmits information with the nodes acting as the initiator and the forwarder, is also known as flying wireless network(WANET), due to their mobile nature and capability of framing a network on the move.

The limitations of the flying wireless network and the improvement in the transmission process of the network subduing the limitations, using various routing protocols are surveyed. Further the analysis representing the efficiency of the protocols are presented in the paper based on the survey.

The paper is organized with the section 2 detailing the limitations of the flying wireless networks, section 3 with the survey of the possible routing solutions that are available, section 4 the analysis of the routing protocols and its efficiency on different performance metrics such as energy consumption, network life time and the quality of service.

\section{Flying Wireless Networks}

The flying wireless networks as the name states are the networks formed on the fly with the available number of devices that exist nearby. The network framed is temporary without infrastructures and equipped with the devices (nodes) with the capability of high mobility [14].So the devices employed are certain of leaving the network at any time without a prior information. This mobile nature of the nodes makes the network unacquainted with its topology, and breaks the connectivity of the network making the location prediction difficult. The link stability of the network is also another challenge in the flying wireless network as they operate with the limited battery power of the nodes. Further the heterogeneity of the nodes, project limitations in the terms of processing capabilities and the storage capacities. The above characteristics of the network bring in the challenges for the process of transmission thus 
J. Sustain. Wireless Syst.

Vol.01/ No. 03

Pages: $148-160$

http://irojournals.com/irosws/

DOI: https://doi.org/10.36548/jsws.2019.3.002

making it difficult. Some of the characteristics of the flying wireless networks, their causes, the challenges they bring into the network transmission process are tabulated as in the table 1 below.

Table 1: Characteristics of Flying Wireless Networks

\begin{tabular}{|l|l|l|}
\hline Characteristics & Challenges & \multicolumn{1}{|c|}{ causes } \\
\hline Infrastructure less & No control entities & $\begin{array}{l}\text { Error prone, weak signal, covers } \\
\text { small geographical area, less } \\
\text { secure,unpredictable decrease in } \\
\text { interconnectivity , de } \\
\text { throughput, decreased reliability }\end{array}$ \\
\hline Mobile & Dynamic topological changes & $\begin{array}{l}\text { Frequent topological changes, } \\
\text { coverage problems, throughput } \\
\text { deteriorates, delay due to re- } \\
\text { routing. }\end{array}$ \\
\hline Battery Powered & Limited power availability & $\begin{array}{l}\text { Node failure, link instability, } \\
\text { interruption in the transmission, } \\
\text { frequent path break, failure in the } \\
\text { packet conveyance, affects } \\
\text { throughput, increases delay }\end{array}$ \\
\hline Resource Constraints & $\begin{array}{l}\text { Limited storage and the processing } \\
\text { capability }\end{array}$ & $\begin{array}{l}\text { Low memory, low processing } \\
\text { capability, limited bandwidth } \\
\text { capability, throughput is affected }\end{array}$ \\
\hline Hidden Node Problem & $\begin{array}{l}\text { Difficulty in next node prediction } \\
\text { co-channel interference }\end{array}$ & $\begin{array}{l}\text { Collision, difficulty in path } \\
\text { prediction, latency, throughput } \\
\text { reduction }\end{array}$ \\
\hline Availability & $\begin{array}{l}\text { Nodes may remain in a network or } \\
\text { leave the network any time, } \\
\text { Propagation delay if not properly } \\
\text { deployed, minimized throughput }\end{array}$ \\
\hline
\end{tabular}

The Table 1 shows the limitation of the flying wireless network, the causes for the limitations and the challenges they bring into the network in the process of the transmission. The routing protocols designed to overcome the challenges in the transmission process addressing the limitations of the network is presented in the next section that includes the survey of the routing protocols for the flying wireless networks. The characteristics of the flying wireless networks were obtained from the survey conducted through the [1], [2], [3], [10] and [48] 
J. Sustain. Wireless Syst.

Vol.01/ No. 03

Pages: $148-160$

http://irojournals.com/irosws/

DOI: https://doi.org/10.36548/jsws.2019.3.002

\section{Routing Protocols for the Flying Wireless Networks}

The Routing protocol is a convention or a standard that allows the nodes (Data Points) to decide on the perfect route to convey the data packets between the source and the target nodes, using the other nodes within their path as forwarders. The routing protocols are essential to a network to increase its performance on the delay incurred, energy consumed, channel overhead, bandwidth utilization, throughput achieved and the packet delivered. So the routing protocols for the flying wireless network help in improving its network performance subduing its limitations and the challenges in the transmission process. The survey on the routing protocols for the flying wireless network is detailed in the table 2 below. The routing protocols developed are categorized as flat (reactive and proactive), hybrid, hierarchical, location based, and further as heuristic and metaheuristic based routing on the grounds of the methodologies used in it . The table 2 below lists the survey on the routing protocols to improve the performance of the flying wireless network in terms of energy, reliability, connectivity and susceptibility to the topological changes [1], increasing the lifetime of the network.

The paper proceeds the survey with the aim of having a prolonged network longevity for the flying wireless network. The table below gives the researches done to improve the performance metrics of the flying wireless network achieving an enhancement in the lifespan of the network.

Table 2: Routing Protocols for Flying Wireless Networks

\begin{tabular}{|c|c|c|c|}
\hline Aim & Protocols & Research's surveyed & Proficiency \\
\hline \multirow{2}{*}{$\begin{array}{l}\text { To have a routing } \\
\text { protocol with the energy } \\
\text { conservation so as to } \\
\text { have enhanced network } \\
\text { lifespan and quality of } \\
\text { service }\end{array}$} & $\begin{array}{l}\text { Flat (Pro-active and } \\
\text { Reactive ) }\end{array}$ & $\begin{array}{l}\text { The capabilities of flat } \\
\text { protocols[3], CAMP Protocol } \\
\text { with proactive routing [4], } \\
\text { secure AODV [7], New } \\
\text { improved AODV[14], AODV } \\
\text { and DSDV [19] }\end{array}$ & $\begin{array}{l}\text { Capabilities of flat } \\
\text { routing protocols[3] } \\
\text { Uniform energy } \\
\text { depletion, prolonged } \\
\text { network lifetime[4], } \\
\text { secure against the } \\
\text { attacks[7], stable links, } \\
\text { ruptured link prediction } \\
\text { [14] provides with the } \\
\text { stable link [19] }\end{array}$ \\
\hline & $\begin{array}{l}\text { Hybrid (Proactive } \\
\text { +Reactive ) }\end{array}$ & $\begin{array}{l}\text { Taxonomy and the efficiency } \\
\text { of the hybrid routing [10], } \\
\text { hybrid routing [11] ICA and } \\
\text { RBF [16] RCA-HDRP[17], } \\
\text { HYB-TGR[20], LB+HRP[47] }\end{array}$ & $\begin{array}{l}\text { Energy efficient, reliable, } \\
\text { fault tolerant } \\
\text { [10][11],robust and } \\
\text { scalable, provides stable } \\
\text { link against the high } \\
\text { mobility [16], effective } \\
\text { path selection [17] }\end{array}$ \\
\hline
\end{tabular}


J. Sustain. Wireless Syst.

Vol.01/ No. 03

Pages: $148-160$

http://irojournals.com/irosws/

DOI: https://doi.org/10.36548/jsws.2019.3.002

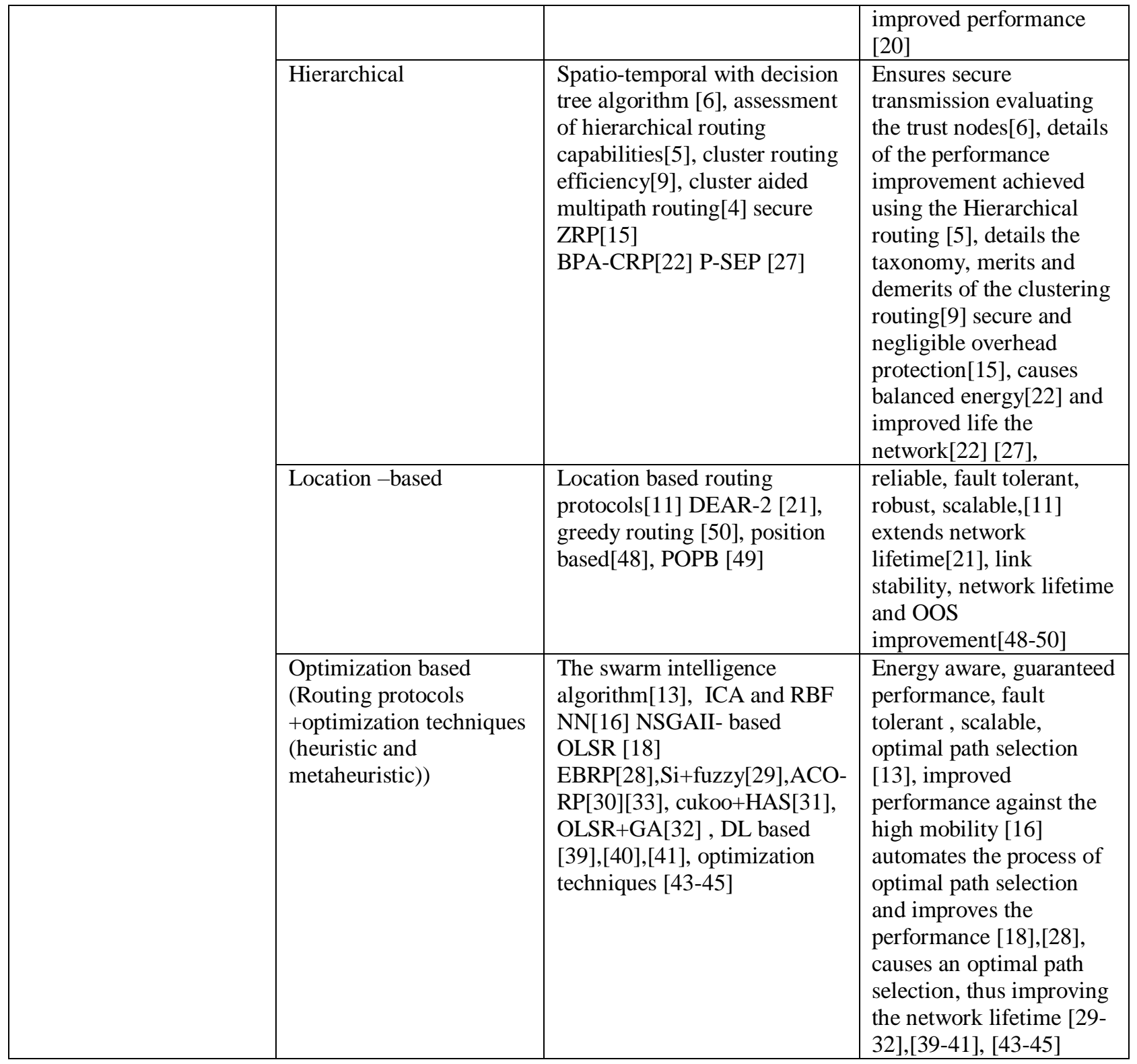

\section{Analysis of the Routing Protocols}

The routing protocols survey based on the categories of the methodologies used for the routing is analyzed for its performance using the Network Simulator -2. By considering the number of nodes that varies between 100 to 200, over a simulation area of $2500 * 40 \mathrm{~m}$ and the simulation time of 100 seconds. The packets size ranges to 1024 bytes with the packet data rate of one packet per second. The routing protocols surveyed are simulated using the network simulator to know the lifetime of the network, the energy consumption and the quality of service. As the network 
J. Sustain. Wireless Syst.

Vol.01/ No. 03

Pages: $148-160$

http://irojournals.com/irosws/

DOI: https://doi.org/10.36548/jsws.2019.3.002

lifetime decides the capability of the network in continuing the transmission process and the energy consumption is very essential for the extending the network lifetime of the nodes thus maintaining the stableness of the link. The QOS of the network is measured to ensure the delay less successful transmission through the network that is mobile in nature.

The Fig 1 shows the energy consumption of the routing protocols, which are based on the methodologies such as flat routing protocols (FRP), hierarchical (HRP), hybrid (HDRP), location based (LBRP) and the routing involving optimization (heuristic and metaheuristic)(RPWO), the simulation results evinces that the routing protocols including the optimization process that enables in finding the optimal path using the metaheuristic and the heuristic shows higher energy conservation than other methods that are reactive, hierarchical, hybrid, proactive and location based. The other methods have difficulties in identifying the optimal path, so the energy is wasted in identifying the path that are liable to be failed. But the heuristic and the metaheuristic method identifies the optimal path easily and proceeds with the transmission without much energy consumption.

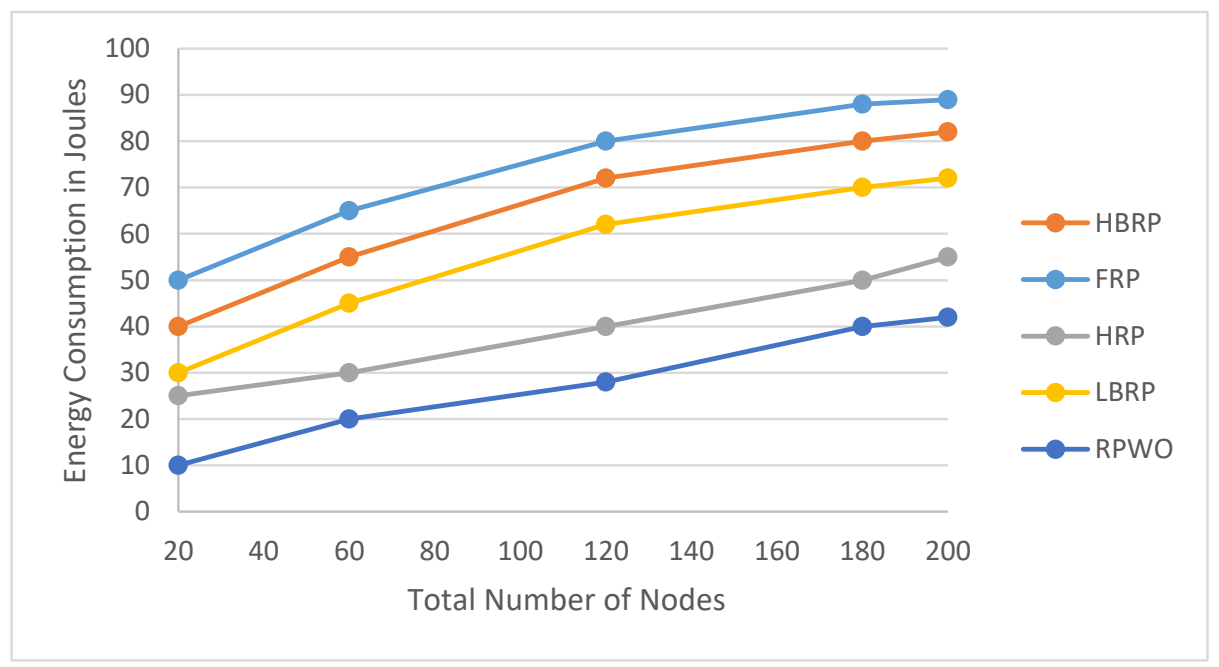

Fig 1: Energy Consumption

The fig 2 shows the simulation results for the lifetime of the flying wireless network applying the various protocols surveyed, the simulation result shows that all routing protocols with the optimization techniques allows the flying wireless network to have an extended lifetime than the other routing protocols that are reactive, proactive, hybrid hierarchical and location based. The comparison shows that the routing protocols with the optimization gives 35\%

ISSN: $2582-3$

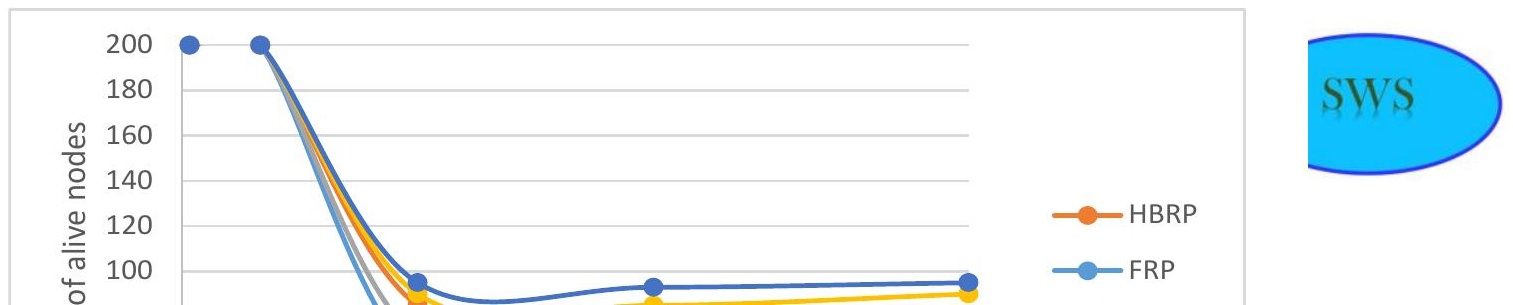


J. Sustain. Wireless Syst.

Vol.01/ No. 03

Pages: $148-160$

http://irojournals.com/irosws/

DOI: https://doi.org/10.36548/jsws.2019.3.002

better results than the proactive and the on-demand methods and $26 \%$ better results than the hybrid and the location based.

The Fig 3 shows the simulation results that details the quality of service provided by each routing protocol, the routing protocol with optimization in the results is evinced to have higher quality of service than the others, the RPWO shows $42.3 \%$ higher quality of service than the FRP and $15.2 \%$ improvement than the HRP and $30 \%$ better quality than the hybrid and $37.08 \%$ better quality then the location based routing protocol.

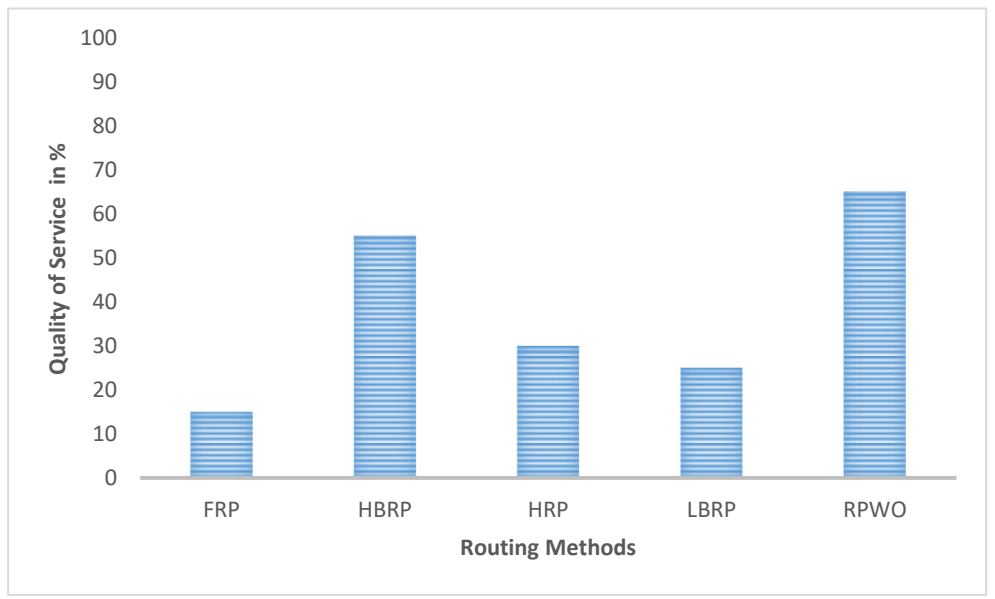

Fig 3 Quality of Service 
J. Sustain. Wireless Syst.

Vol.01/ No. 03

Pages: $148-160$

http://irojournals.com/irosws/

DOI: https://doi.org/10.36548/jsws.2019.3.002

Thus the analysis for the performance of the routing protocols for the flying wireless networks, by simulating through the network simulator-2 evinces that the routing protocol along with the optimization techniques shows higher efficiency then the flat, hybrid, hierarchical and the location based routing. Further the experiment analysis on the delay computations (D) and the time complexity (T) for the routing algorithms surveyed in shown in the table 3 below.

\begin{tabular}{|c|c|c|c|c|c|c|c|c|c|c|}
\hline Rounds & \multicolumn{2}{|c|}{ Frotocols } & \multicolumn{2}{c|}{ HBRP } & \multicolumn{2}{c|}{ LBRP } & \multicolumn{2}{c|}{ HRP } & \multicolumn{2}{c|}{ RPWO } \\
\cline { 2 - 10 } & $\mathrm{T}$ & $\mathrm{D}$ & $\mathrm{T}$ & $\mathrm{D}$ & $\mathrm{T}$ & $\mathrm{D}$ & $\mathrm{T}$ & $\mathrm{D}$ & $\mathrm{T}$ & $\mathrm{D}$ \\
\hline 1 & 4.15 & 1.2467 & 3.25 & 1.2307 & 3.58 & 1.2301 & 4.17 & 1.0467 & 2.0 & 1.1227 \\
\hline 50 & 4.25 & 1.2561 & 3.54 & 1.2326 & 3.16 & 1.2035 & 3.46 & 1.1461 & 2.18 & 1.0567 \\
\hline 100 & 4.08 & 1.3670 & 3.27 & 1.1735 & 3.18 & 1.2274 & 3.10 & 1.1370 & 2.30 & 1.0246 \\
\hline 500 & 4.11 & 1.3237 & 3.10 & 1.2032 & 3.09 & 1.1448 & 3.03 & 1.0237 & 2.13 & 0.8754 \\
\hline 1000 & 4.02 & 1.1568 & 3.05 & 1.0185 & 3.02 & 1.0290 & 2.59 & 0.9568 & 2.08 & 0.8267 \\
\hline
\end{tabular}

Table 3 Time Complexity and the Delay Computation of the Protocols

The Table 3 show the time complexity and the delay calculations for the routing algorithms under the methods FRP, HRP, HBRP, LBRP, and Routing protocol with optimization, the results obtained from the experiment shows that the Routing protocols integrated with the optimization techniques show better results compared to the other methods of routing, thus offering a better quality of service. So the survey conducted helps in identifying the routing protocols that enables in having an optimal path selection, reducing the time, energy consumption, delay and prolonging the network longevity. Further the elite optimized method of routing identification within the routing protocols integrated with the optimization technique is to be continued in the future.

\section{Conclusion}

The paper details the study on the routing protocols available for the flying wireless network, as the routing protocol ensures the performance improvement of the network addressing its limitations and subduing its challenges, the routing protocol enables the transmission process to take place establishing the optimal path between the source and destination eluding the delay, overhead, maximum energy utilization and the packet delivery losses. So the paper proceeded with the limitation of the flying wireless network and then continued with the survey on the various 
J. Sustain. Wireless Syst.

Vol.01/ No. 03

Pages: $148-160$

http://irojournals.com/irosws/

DOI: https://doi.org/10.36548/jsws.2019.3.002

routing protocols that are developed for the flying wireless network. Further the performance analysis was done for the various protocols based on the metrics such as network lifetime, energy consumption and the quality of service to evince the optimal routing method and it was identified that he routing protocols along with the optimization techniques were better compared to the other methods as they involved the heuristic and the metaheuristic process in the selection of the optimal path that reduced energy and improved the network lifetime. So the survey done on the paper would enable the development of the routing protocol with the optimization techniques for the flying wireless network, further the paper is to proceed with the study on the able optimization technique that identifies the elite path for routing enable a considerable reduction in the energy and achieving heightened lifespan for the network.

\section{References}

[1] Jahir, Yasmin, Mohammed Atiquzzaman, Hazem Refai, Anirudh Paranjothi, and Peter G. LoPresti. "Routing protocols and architecture for disaster area network: A survey." Ad Hoc Networks 82 (2019): 114.

[2] Bhushan, Bharat, and G. Sahoo. "Routing protocols in wireless sensor networks." In Computational Intelligence in Sensor Networks, pp. 215-248. Springer, Berlin, Heidelberg, 2019.

[3] Saudi, Nur Amirah Mohd, Mohamad Asrol Arshad, Alya Geogiana Buja, Ahmad Firdaus Ahmad Fadzil, and Raihana Md Saidi. "Mobile Ad-Hoc Network (MANET) Routing Protocols: A Performance Assessment." In Proceedings of the Third International Conference on Computing, Mathematics and Statistics (iCMS2017), pp. 53-59. Springer, Singapore, 2019.

[4] Sajwan, Mohit, Devashish Gosain, and Ajay K. Sharma. "CAMP: cluster aided multi-path routing protocol for wireless sensor networks." Wireless Networks 25, no. 5 (2019): 2603-2620.

[5] Saini, Trilok Kumar, and Subhash C. Sharma. "Prominent unicast routing protocols for Mobile Ad hoc Networks: Criterion, classification, and key attributes." Ad Hoc Networks89 (2019): 58-77.

[6] Selvi, M., K. Thangaramya, Sannasi Ganapathy, Kanagasabai Kulothungan, H. Khannah Nehemiah, and Arputharaj Kannan. "An Energy Aware Trust Based Secure Routing Algorithm for Effective Communication in Wireless Sensor Networks." Wireless Personal Communications 105, no. 4 (2019): 1475-1490.

[7] Soni, Megha, and Brijendra Kumar Joshi. "Security Assessment of SAODV Protocols in Mobile Ad hoc Networks." In Data Science and Big Data Analytics, pp. 347-355. Springer, Singapore, 2019.

[8] Ahmad, Sabbagh Amani, and Maxim Shcherbakov. "A Survey on Routing Protocols in Vehicular Adhoc Networks." In 2018 9th International Conference on Information, Intelligence, Systems and Applications (IISA), pp. 1-8. IEEE, 2018. 
J. Sustain. Wireless Syst.

Vol.01/ No. 03

Pages: 148-160

http://irojournals.com/irosws/

DOI: https://doi.org/10.36548/jsws.2019.3.002

[9] Singh, Santar Pal, and S. C. Sharma. "A survey on cluster based routing protocols in wireless sensor networks." Procedia computer science 45 (2015): 687-695.

[10] Walikar, Gyanappa A., and Rajashekar C. Biradar. "A survey on hybrid routing mechanisms in mobile ad hoc networks." Journal of Network and Computer Applications 77 (2017): 48-63.

[11] Mahmood, Baban A., and D. Manivannan. "Position based and hybrid routing protocols for mobile ad hoc networks: a survey." Wireless Personal Communications 83, no. 2 (2015): 1009-1033.

[12] Tareque, Md Hasan, Md Shohrab Hossain, and Mohammed Atiquzzaman. "On the routing in flying ad hoc networks." In 2015 Federated Conference on Computer Science and Information Systems (FedCSIS), pp. 19. IEEE, 2015.

[13] Gui, Tina, Christopher Ma, Feng Wang, and Dawn E. Wilkins. "Survey on swarm intelligence based routing protocols for wireless sensor networks: An extensive study." In 2016 IEEE International Conference on Industrial Technology (ICIT), pp. 1944-1949. IEEE, 2016.

[14] Saoud, Bilal, and Abdelouahab Moussaoui. "New Routing Protocol in Ad Hoc Networks." In International Conference on Computer Networks and Communication Technologies, pp. 443-452. Springer, Singapore, 2019.

[15] Trivedi, Munesh C., Sakshi Yadav, and Vikash Kumar Singh. "Securing ZRP Routing Protocol Against DDoS Attack in Mobile Ad Hoc Network." In Advances in Data and Information Sciences, pp. 387-396. Springer, Singapore, 2019.

[16] Mohammadnezhad, Mojtaba, and Ali Ghaffari. "Hybrid routing scheme using imperialist competitive algorithm and RBF neural networks for VANETs." Wireless Networks 25, no. 5 (2019): 2831-2849.

[17] Chai, Yuan, and Xiao-Jun Zeng. "Regional condition-aware hybrid routing protocol for hybrid wireless mesh network." Computer Networks 148 (2019): 120-128.

[18]Harrag, Nassir, Allaoua Refoufi, and Abdelghani Harrag. "New NSGA-II-based OLSR self-organized routing protocol for mobile ad hoc networks." Journal of Ambient Intelligence and Humanized Computing 10, no. 4 (2019): 1339-1359.

[19] Lavanya, P., V. S. K. Reddy, and A. Mallikarjuna Prasad. "Simulation and QoS Metrics Comparison of Routing Protocols for Mobile Ad hoc Networks Using Network Simulator." In Microelectronics, Electromagnetics and Telecommunications, pp. 567-575. Springer, Singapore, 2019.

[20] Arianmehr, Shahrbanou, and Mohammad Ali Jabraeil Jamali. "HybTGR: a hybrid routing protocol based on topological and geographical information in vehicular ad hoc networks." Journal of Ambient Intelligence and Humanized Computing(2019): 1-13.

[21] Hassan, Muhammad Umair, Muhammad Shahzaib, Kamran Shaukat, Syed Nakhshab Hussain, Muhammad Mubashir, Saad Karim, and Muhammad Ahmad Shabir. "DEAR-2: An Energy-Aware Routing Protocol with Guaranteed Delivery in Wireless Ad-hoc Networks." In Recent Trends and Advances in Wireless and IoT-enabled Networks, pp. 215-224. Springer, Cham, 2019. 
J. Sustain. Wireless Syst.

Vol.01/ No. 03

Pages: 148-160

http://irojournals.com/irosws/

DOI: https://doi.org/10.36548/jsws.2019.3.002

[22] Darabkh, Khalid A., Mohammad Z. El-Yabroudi, and Ali H. El-Mousa. "BPA-CRP: A balanced poweraware clustering and routing protocol for wireless sensor networks." Ad Hoc Networks 82 (2019): 155-171.

[23] Reddy, G. Karthik, and L. Nirmala Devi. "A Review on Clustering Protocols with Energy heterogeneity in Wireless Sensor Networks." In 2018 International Conference on Communication, Computing and Internet of Things (IC3IoT), pp. 243-246. IEEE, 2018.

[24] Guleria, Kalpna, and Anil Kumar Verma. "Comprehensive review for energy efficient hierarchical routing protocols on wireless sensor networks." Wireless Networks 25, no. 3 (2019): 1159-1183.

[25]Dhingra, Madhavi, S. C. Jain, and Rakesh Singh Jadon. "Performance Comparison of LANMAR and AODV in Heterogenous Wireless Ad-hoc Network." In Emerging Trends in Expert Applications and Security, pp. 125-132. Springer, Singapore, 2019.

[26]Li, Fan, Xiaoyu Song, Huijie Chen, Xin Li, and Yu Wang. "Hierarchical Routing for Vehicular Ad Hoc Networks via Reinforcement Learning." IEEE Transactions on Vehicular Technology 68, no. 2 (2018): 1852-1865.

[27] Naranjo, Paola G. Vinueza, Mohammad Shojafar, Habib Mostafaei, Zahra Pooranian, and Enzo Baccarelli. "P-SEP: A prolong stable election routing algorithm for energy-limited heterogeneous fog-supported wireless sensor networks." The Journal of Supercomputing 73, no. 2 (2017): 733-755.

[28] Shokouhifar, Mohammad, and Ali Jalali. "A new evolutionary based application specific routing protocol for clustered wireless sensor networks." AEU-International Journal of Electronics and Communications 69, no. 1 (2015): 432-441.

[29]Zahedi, Zeynab Molay, Reza Akbari, Mohammad Shokouhifar, Farshad Safaei, and Ali Jalali. "Swarm intelligence based fuzzy routing protocol for clustered wireless sensor networks." Expert Systems with Applications 55 (2016): 313-328.

[30] Mohajerani, Abdolreza, and Davood Gharavian. "An ant colony optimization based routing algorithm for extending network lifetime in wireless sensor networks." Wireless Networks 22, no. 8 (2016): 2637-2647.

[31] Gupta, Govind P., and Sonu Jha. "Integrated clustering and routing protocol for wireless sensor networks using Cuckoo and Harmony Search based metaheuristic techniques." Engineering Applications of Artificial Intelligence 68 (2018): 101-109.

[32]Zhang, De-gan, Ting Zhang, Yue Dong, Xiao-huan Liu, Yu-ya Cui, and De-xin Zhao. "Novel optimized link state routing protocol based on quantum genetic strategy for mobile learning." Journal of Network and Computer Applications 122 (2018): 37-49.

[33] Arjunan, Sariga, and Pothula Sujatha. "Lifetime maximization of wireless sensor network using fuzzy based unequal clustering and ACO based routing hybrid protocol." Applied Intelligence 48, no. 8 (2018): 2229-2246.

[34]Elhabyan, Riham, Wei Shi, and Marc St-Hilaire. "A Pareto optimization-based approach to clustering and routing in Wireless Sensor Networks." Journal of Network and Computer Applications 114 (2018): 57-69. 
J. Sustain. Wireless Syst.

Vol.01/ No. 03

Pages: 148-160

http://irojournals.com/irosws/

DOI: https://doi.org/10.36548/jsws.2019.3.002

[35]Logambigai, R., Sannasi Ganapathy, and Arputharaj Kannan. "Energy-efficient grid-based routing algorithm using intelligent fuzzy rules for wireless sensor networks." Computers \& Electrical Engineering 68 (2018): 62-75.

[36] Kong, Lingping, Jeng-Shyang Pan, Václav Snášel, Pei-Wei Tsai, and Tien-Wen Sung. "An energy-aware routing protocol for wireless sensor network based on genetic algorithm." Telecommunication Systems 67, no. 3 (2018): 451-463.

[37] Mao, Bomin, Fengxiao Tang, Zubair Md Fadlullah, Nei Kato, Osamu Akashi, Takeru Inoue, and Kimihiro Mizutani. "A novel non-supervised deep-learning-based network traffic control method for software defined wireless networks." IEEE Wireless Communications 25, no. 4 (2018): 74-81.

[38] Fu, Yu, Sen Wang, Cheng-Xiang Wang, Xuemin Hong, and Stephen McLaughlin. "Artificial intelligence to manage network traffic of 5G wireless networks." IEEE Network 32, no. 6 (2018): 58-64.

[39] Lu, Jiayi, Luanye Feng, Jun Yang, Mohammad Mehedi Hassan, Abdulhameed Alelaiwi, and Iztok Humar. "Artificial agent: The fusion of artificial intelligence and a mobile agent for energy-efficient traffic control in wireless sensor networks." Future Generation Computer Systems 95 (2019): 45-51.

[40] Aslam, Nelofar, Kewen Xia, and Muhammad Usman Hadi. "Optimal Wireless Charging Inclusive of Intellectual Routing based on SARSA Learning in Renewable Wireless Sensor Networks." IEEE Sensors Journal (2019).

[41] Tang, Fengxiao, Bomin Mao, Zubair Md Fadlullah, Nei Kato, Osamu Akashi, Takeru Inoue, and Kimihiro Mizutani. "On removing routing protocol from future wireless networks: A real-time deep learning approach for intelligent traffic control." IEEE Wireless Communications 25, no. 1 (2017): 154-160.

[42] Mao, Qian, Fei Hu, and Qi Hao. "Deep learning for intelligent wireless networks: A comprehensive survey." IEEE Communications Surveys \& Tutorials 20, no. 4 (2018): 2595-2621.

[43] Brar, Gurbinder Singh, Shalli Rani, Vinay Chopra, Rahul Malhotra, Houbing Song, and Syed Hassan Ahmed. "Energy efficient direction-based PDORP routing protocol for WSN." IEEE access 4 (2016): 3182-3194.

[44] Sudhakar, T., and H. Hannah Inbarani. "Intelligent Path Selection in Wireless Networks using Jaya Optimization." Procedia computer science 133 (2018): 976-983.

[45] Sun, Ziwen, Min Wei, Zhiwei Zhang, and Gang Qu. "Secure Routing Protocol based on Multi-objective Ant-colony-optimization for wireless sensor networks." Applied Soft Computing 77 (2019): 366-375.

[46] Randhawa, Sukhchandan, and Sushma Jain. "MLBC: Multi-objective Load Balancing Clustering technique in Wireless Sensor Networks." Applied Soft Computing 74 (2019): 66-89.

[47] Vahabi, Shahrokh, Mohammadreza Eslaminejad, and Seyed Ebrahim Dashti. "Integration of geographic and hierarchical routing protocols for energy saving in wireless sensor networks with mobile sink." Wireless Networks 25, no. 5 (2019): 2953-2961. 
J. Sustain. Wireless Syst.

Vol.01/ No. 03

Pages: $148-160$

http://irojournals.com/irosws/

DOI: https://doi.org/10.36548/jsws.2019.3.002

[48] Liu, Jianqi, Jiafu Wan, Qinruo Wang, Pan Deng, Keliang Zhou, and Yupeng Qiao. "A survey on positionbased routing for vehicular ad hoc networks." Telecommunication Systems 62, no. 1 (2016): 15-30.

[49] Rajesh, M., and J. M. Gnanasekar. "Path observation based physical routing protocol for wireless ad hoc networks." Wireless Personal Communications 97, no. 1 (2017): 1267-1289.

[50] Liu, Jinwei, Haiying Shen, Lei Yu, Husnu Saner Narman, Jiannan Zhai, Jason O. Hallstrom, and Yangyang He. "Characterizing data deliverability of greedy routing in wireless sensor networks." IEEE Transactions on Mobile Computing17, no. 3 (2017): 543-559.

[51] Anasane, Aboli Arun, and Rachana Anil Satao. "A survey on various multipath routing protocols in wireless sensor networks." Procedia Computer Science 79 (2016): 610-615. 